\title{
Monoamine Oxidase and Semi Carbazide-Sensitive Amine Oxidase in Pathogenetic Mechanisms of First Episode of Schizophrenia
}

\author{
Uzbekov $\mathbf{M}^{1 *}$, Brilliantova $\mathrm{V}^{2}$ and Shikhov $\mathrm{S}^{3}$ \\ ${ }^{1}$ Head of the Laboratory of Brain Pathology, Moscow Research Institute of Psychiatry, Russia \\ ${ }^{2} J$ unior researcher, Laboratory of Brain Pathology, Moscow Research Institute of Psychiatry, Russia \\ ${ }^{3}$ Senior researcher, Laboratory of Brain Pathology, Moscow Research Institute of Psychiatry, Russia \\ *Corresponding author: Uzbekov M, Professor, Head of the Laboratory of Brain Pathology, Moscow Research Institute of Psychiatry, \\ Russia
}

\section{ARTICLE INFO}

Received: 幽 November 14, 2019

Published: 幽 November 21, 2019
ABSTRACT

Abbreviations: FES: First Episode of Schizophrenia; MAO: Monoamine Oxidase; SSAO: Semi Carbazide-Sensitive Amine Oxidase; SAO: Sensitive Amine Oxidase

Citation: Uzbekov M, Brilliantova V, Shikhov S. Monoamine Oxidase and Semi Carbazide-Sensitive Amine Oxidase in Pathogenetic Mechanisms of First Episode of Schizophrenia. Biomed J Sci \& Tech Res 23(1)-2019. BJSTR. MS.ID.003856.

\section{Introduction}

Therapy of patients with first episode of schizophrenia (FES) is a complicated task for psychiatrists. Early diagnosis and effective therapy are key steps for achieving the long-term positive outcomes among such kind of patients. It seems to be generally accepted that patients suffering from a FES tend to be more responsive to antipsychotic treatment than patients with chronic schizophrenia [1]. Effective treatment of FES may decrease the number of relapses of the disease and increase the level of social functioning and improve their quality of life. Despite of intensive studies, the pathogenetic mechanisms of the first episode of schizophrenia are poorly studied. Hence, it is very important to study the pathophysiological mechanisms of first episode of schizophrenia. The current diagnosis of schizophrenia remains subjective for many reasons and one of them is the lack of biological markers of the disease. The psychiatric clinic needs diagnostic, differentia-diagnostic and prognostic tests and tests for the evaluation of the efficacy of psychopharmacotherapy, psychotherapy and other types of interventions. The identification of biological markers of psychosis is a fundamental step towards a better understanding of pathogenetic mechanisms of psychosis and holds the potential for developing more objective testing method [2]. Neurochemical studies help to understand the pathogenetic mechanisms of schizophrenia, including the first episode of the disease, and to create antipsychotic drugs with new characteristics and new targets. The aim of the study was to investigate the activity of platelet monoamine oxidase (MAO) and semi carbazide-sensitive amine oxidase (SSAO) in the blood serum of drug-naïve first episode (FES) schizophrenic patients.

\section{Methods}

This study was performed with 26 patients with FES (11 females and 15 males, average age was28.29.5 years) who were examined with biochemical and clinical methods. All patients gave informed consent on the participation in the study. Investigation was performed in accordance of Helsinki Declaration and on 
conclusion of the local ethical committee of the Institute $(\mathrm{N}$ $43 / 2$ of 27/04/2009). Inclusion criteria were as follows: age > 18 years, first psychotic episode characterized by PANSS score $>60$, schizophrenia was diagnosed according to International Classifications of Diseases, tenth edition, clinical modification (ICD-10-CM) [3], patient had not undergone antipsychotic therapy, and stable somatic health. Exclusion criteria were alcohol or drug abuse, severe somatic diseases, pathologically altered standard biochemical parameters and any therapy by psychotropic drugs before the study. In all cases, it was diagnosed schizophrenia: 21 patients with paranoid schizophrenia (F20.0) and 5 patients with nondifferentiated schizophrenia (F20.3). The severity of the disease was evaluated using the PANSS (Positive and Negative Symptom Scale). The control group included 15 subjects of both sexes who were considered to be healthy people according to clinical and biochemical characteristics.

The biochemical parameters of FES patients were measured at admission before the start of pharmacotherapy. Platelet monoamine oxidase (MAO) and serum semi carbazide-sensitive amine oxidase (SAO) activities were measured by methods described by Voloshina and Moskvitina [4] and Balakleevskii [5], respectively. The data are presented as mean standard deviation. Significance of differences between the parameters of healthy volunteers and patients were evaluated using the Student's t test. The type of interaction of the parameters studied was evaluated using regression and factor analysis. The factor analysis was performed with the use of two methods: the method of principal components and the multiple R-square method. All statistical calculations were performed with the use of Statistics for Windows, version 6.0. Differences were considered as significant at $\mathrm{p}=0.05$.

\section{Results}

The severity of the disease before treatment was75.5 \pm 2.2 points according to the PANSS scale, which corresponds to moderate severity. Before the treatment, patients with FES were characterized by a significant increased activity of platelet MAO (by 107\%, p= 0.01) and decreased SAO activity (by 29\%; p= 0.001 ) in the blood serum, as compared to the control (Table 1). Regression analysis showed a significant correlation between blood parameters, the activity of monoamine oxidase $(p=0.0101)$ and semi carbazide-sensitive amine oxidase $(p=0.0109)$, and clinical index of the disease severity, PANSS. The correlation between MAO and SAO activity was highly significant $(p=0.01)$.The relationship between biochemical variables was evaluated by factor analysis. Factor analysis has shown that MAO, SSAO and PANSS parameters are covered by Factor 1 . It was determined that both methods of extraction (principle components and multiple R-square) gave very similar results.

Table 1: Platelet monoamine oxidase (MAO) and serum semi carbazide-sensitive amine oxidase (SSAO) activities of FES patients and controls.

\begin{tabular}{|c|c|c|}
\hline & $\begin{array}{c}\text { MAO } \\
\text { SSAO }\end{array}$ & $\begin{array}{c}\text { (nmole benzaldehyde/ml serum/h) } \\
\text { (nmole benzaldehyde/mg protein/h) }\end{array}$ \\
\hline Controls $(\mathrm{n}=15)$ & $8.68 \pm 1.65$ & $10.84 \pm 1.65$ \\
\hline Patients $(\mathrm{n}=26)$ & $17.30 \pm 1.72^{*}$ & $8.03 \pm 0.59^{* *}$ \\
\hline
\end{tabular}

Note: * and **, significant difference between FES and controls at $\mathrm{p}=0.01$ and $\mathrm{p}=0.001$, respectively.

\section{Discussion}

Thus, our study showed that FES is characterized by biochemical disturbances: a significant increase in the activity of platelet MAO, which may reflect MAO activity in the brain [6], and a decrease in the SSAO activity. There are very few studies on the state of biochemical processes in patients with FES and the results are very contradictory. On the basis of analysis of our own [7] and literature data, we found an explanation of the fact that the activity of platelet MAO is increased in the patients with FES before the onset of treatment. It is known that dopaminergic neurotransmission is hyperactivated in patients with FES. It can be accompanied by the accumulation of excess of dopamine in the synaptic cleft. Therefore, it is possible to hypothesize that the increase in the MAO activity is a compensatory event, which should lead to a decrease in the dopamine concentration in the synaptic cleft. Semi carbazide-sensitive amine oxidase of blood serum is an enzyme that, in addition to the system of cytochrome P-450, is involved in the oxidation of xenobiotics, including drugs and endogenous substances [8]. The present study is the first to investigate SSAO activity in FES patients. Currently, it is hard to explain the decrease in the SSAO activity in patients with FES. SSAO can convert some endogenous amines, such as methylamine and aminoacetone, into highly toxic compounds - formaldehyde and methylglyoxal, respectively, as well as acrolein that may have negative effects. It is possible to hypothesize that the concentration of toxic metabolites is increased in patients with FES, which promotes an increase in endogenous intoxication. The accumulation of toxic metabolites may trigger a compensatory mechanism of allosteric inhibition, which results in the inhibition of enzyme activity and a decrease in the production of toxic metabolites.

\section{References}

1. Buckley PF, Evans D (2006) First episode schizophrenia - an opportunity for therapeutic intervention. J Postgrad Med 51: 5-19.

2. Jindal RD, Keshavan MS (2008) Neurobiology of the early course of schizophrenia. Expert Rev Neurother 8(7): 1093-1100.

3. (2015) World Health Organization. International Classification of Diseases and Related Problems. (10 th $e d n)$ Clinical Modification, WHO, Geneva, Switzerland. 
4. Voloshina ON, Moskvitina TA (1985) Method of estimation of platelet monoamine oxidase activity. Lab Delo 5: 289-291.

5. Balakleevski AI (1976) Colorimetric method of estimation of serum monoamine oxidase activity. Lab Delo 3: 151-153.

6. Chen K, Wu HF, Shih JC (1993) The deduced amino acid sequences of human platelet and frontal cortex monoamine oxidase B are identical. J Neurochem 61(1): 187-190.

\section{ISSN: 2574-1241}

DOI: 10.26717/BJSTR.2019.23.003856

Uzbekov M. Biomed J Sci \& Tech Res

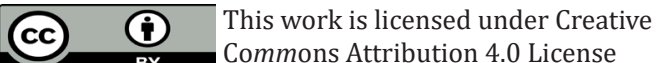

Submission Link: https://biomedres.us/submit-manuscript.php
7. Uzbekov MG, Misionzhnik EYu, Gurovich IY, Shmukler AB (2013) Aspects of metabolic changes in first-episode drug-naïve schizophrenic patients. Acta Neuropsychiatrica 25(5): 268-274.

8. Boomsma F, Bhaggoe UM, van der Houwen AMB, van der Meiracker AH (2003) Plasma semicarbazide-sensitive amine oxidase in human (patho) physiology. Biochim Biophys Acta 1647(1-2): 48-54.

$\begin{array}{ll}\text { BIOMEDICAL } & \text { Assets of Publishing with us } \\ \text { RESEARCHES } & \text { - Global archiving of articles } \\ & \text { - Immediate, unrestricted online access } \\ & \text { - Rigorous Peer Review Process } \\ \end{array}$

